\title{
A Cloud Based Big Data Health-Analytics-as-a-Service Framework to Support Low Resource Setting Neonatal Intensive Care Unit
}

\author{
Meghana Bastwadkar \\ Research Scholar: Computer \\ Science Department, \\ Jain University, Bangalore, India \\ 0091-9448874996 \\ meghana_bastwadkar@yahoo.co.in
}

\author{
Carolyn McGregor \\ Ontario Tech University, Oshawa, \\ Canada \\ University of Technology Sydney, \\ Sydney, Australia \\ +19057218668 \\ c.mcgregor@ieee.org
}

\author{
S. Balaji \\ Centre for Incubation, Innovation, \\ Research and Consultancy \\ Jyothy Institute of Technology, \\ Tataguni, Off Kanakapura Road \\ Bangalore-560082, India \\ 0091-9886055095 \\ drsbalaji@gmail.com
}

\begin{abstract}
Critical care patients are monitored by a range of medical devices collecting high frequency data. New computing frameworks and platforms are being proposed to review and analyze the data in detail. The application of these approaches in a low resource setting is challenged by the approaches used for data acquisition. Software as a Service (SaaS) is a form of cloud computing where a cloudbased software application enables the storage, analysis and visualization of data within the cloud. A subset of SaaS is Health Analytics as a Service (HAaaS), which provides software to support health analytics in the cloud. The objective of this study is to design, implement, and demonstrate an extendable big-data compatible HAaaS framework that offers both real-time and retrospective analysis where data acquisition is not tightly coupled. A data warehousing framework is presented to facilitate analysis within a low resource setting. The framework has been instantiated in the Artemis platform within the context of the Belgaum Children Hospital $(\mathrm{BCH})$ case study. Initial end-to-end testing with the Nellcor monitor (bedside monitor at $\mathrm{BCH}$ ), which was not connected to any human, was completed. This testing confirms the functionality of the new Artemis cloud instance to receive data from test device using an alternate data acquisition approach.
\end{abstract}

\section{CCS Concepts}

Applied Computing $\rightarrow$ Health Informatics, Applied Computing $\rightarrow$ Health Information Systems

\section{Keywords}

premature infants; physiological data; decision support system; analytics-as-a-service; cloud computing; big data, health informatics; real-time analytics; retrospective analysis.

\section{INTRODUCTION}

Critical patients are cared for in intensive care units (ICUs). Infants born premature or ill at term are monitored and cared for in neonatal intensive care units (NICUs). These infants are at risk of death from infections or other complications coupled with the risk of developing lifelong morbidities such as blindness, permanent lung damage and permanent intestine damage to name a few. For this reason, in the neonatal intensive care unit, decision making is a very complicated and critical task. Healthcare providers are concerned with allocating medical resources and treatment according to the baby's medical condition. However, healthcare providers must be cognizant regarding the changes and progression the baby is experiencing as it develops. Previous research has recognized the great potential in using high speed physiological data to screen for changes in health conditions that represent pathophysiologies for a range of conditions that these infants could develop [1]. In addition, translation of the clinical research results to new treatment guidelines has great potential that has not been able to be realized.

As noted in [2], information technology to derive knowledge from this high frequency data in Indian Pediatric Intensive Care Units (PICUs) and NICUs remains a challenge. Like other country practices, patients in India are attached to a lot of medical devices and these medical devices display a lot of data at a high frequency. However, as a result of low resource settings in India, there is no consistency in both care practices and the use of medical devices. Within rural NICUs, patients are often transported onto tertiary CCUs for monitoring by onsite intensivists. There is a lack of expertise available with robust and specialized intensivists setup whilst supporting patients in rural and remote CCUs [3]. For this reason, none of this data is used for decision making by the healthcare professionals. Also, output from these bedside devices is often proprietary and requires commercial data acquisition components.

The Neo device is built on a cost-effective Internet of Things platform that enables vital data acquisition in real-time from devices connected to newborns in NICUs and enables that data to be sent in real-time to the cloud-based big data platform called integrated NICU [4]. However, this involves an integration cost. These, commercial data acquisition components are costly and as a result, their use in low resource settings is not plausible.

Big data analytics frameworks and platforms have been proposed to provide clinical decision support using the data from the bedside devices. These frameworks and platforms for big data analytics within a data warehouse paradigm have the components tightly coupled within the framework and platform. Data acquisition within the Spark framework is within Spark and the data acquisition component of the Artemis framework platform is within Artemis [4]. This means that the data acquisition component of these frameworks is tightly coupled with the other components of the 
clinical decision support system (CDSS). Within Neo device the analytics module takes time series physiological data persisted by a cloud data receiver as input and calculates the probability of a patient being in diseased state [4]. This implies that the data acquisition and data analysis are part of the same system and hence tightly coupled. Artemis has already been deployed within the NICUs of healthcare facilities in Canada and US using HAaaS paradigm [5]. However, the current approach for the HAaaS model involves setting up expensive components within the hospital to collect data from bedside medical devices to forward it to the HAaaS components located offsite.

In this paper, we present a framework for providing HAaaS for both real-time and retrospective analysis where the data acquisition and data transmission components are decoupled from the remaining components in the data warehouse framework for supporting CDSS. This framework is instantiated within a client providing the functionality required for data acquisition and data transmission and a HAaaS platform that is an extended version of the Artemis platform that contains an adaptive API to receive the data. This instantiation is demonstrated within the context of its ability to provide CDSS for the neonatal intensive care unit at Belgaum Children Hospital in Belgaum, India. The $\mathrm{BCH}$ case study research is targeted for low resource setting environment. This paper is an extension of McGregor's prior HAaaS based approach for Artemis allowing functional decoupling of components within the framework [5]. In this paper, we detail the overall case study research plan and provide results from the initial end to end connection testing between the Nellcor 595 pulse oximeters (bedside devices) that are used within BCH NICU and an Artemis cloud instance provisioned at the Centre for Advanced Computing, Queen's University as a component of Compute Ontario infrastructure to demonstrate functional decoupling of components within the proposed big data analytics based framework.

Artemis and Artemis Cloud are first introduced. The proposed extension to enable the functional decoupling is then introduced. Load estimates based on the NICU at Belgaum Children Hospital in Belgaum, India are then presented followed by a section that details the results of testing of the architecture.

\section{LITERATURE REVIEW}

\subsection{Artemis Framework}

This section provides a summary of the Artemis architecture and its various applications. Health care providers still look at clinical information being qualitative rather than quantitative though there has been a shift from paper to electronic because up to now there are no tools, techniques and policies for analysis of complex, highfrequency, physiological data streams. Artemis is an online health analytics platform that can provide high speed physiological data along with electronic health record data and can be used for clinical decision support in neonatal intensive care. As shown in Figure 1, physiological data streams from medical devices together with clinical information are given as input to the data acquisition component of the Artemis platform. Data is processed in real time by the Online Analysis component which employs IBM's Infosphere Streams middleware system. The Data Persistency component of the platform stores both original data along with newly generated analytics. The Knowledge Extraction component enables secondary use of data exploration at a population or individual level and enables the discovery of new pathophysiologies of disease and various other conditions that the neonatal infants can develop such as early onset neonatal sepsis
(EONS), late onset neonatal sepsis (LONS), retinopathy of prematurity (RoP) and anaemia of prematurity [6]. The Online Analytics and Knowledge Extraction components perform the function of real-time and retrospective data analytics, respectively, for the Data Analytics component of a data warehouse structure. The Knowledge Extraction component utilizes a temporal abstraction based deep learning artificial intelligence data mining component created by McGregor known as Multidimensional Service Based Temporal Data Mining $\left(\mathrm{STDM}_{0}{ }^{\mathrm{n}}\right)$ [7]. When a physiological data behaviour is found to be significantly associated with a given neonatal condition through null hypothesis testing, new real-time algorithms are deployed into the Online Analytics component to watch for those physiological behaviours in real-time by the (Re)Deployment component. This component also redeploys enhanced versions of existing algorithms over time.

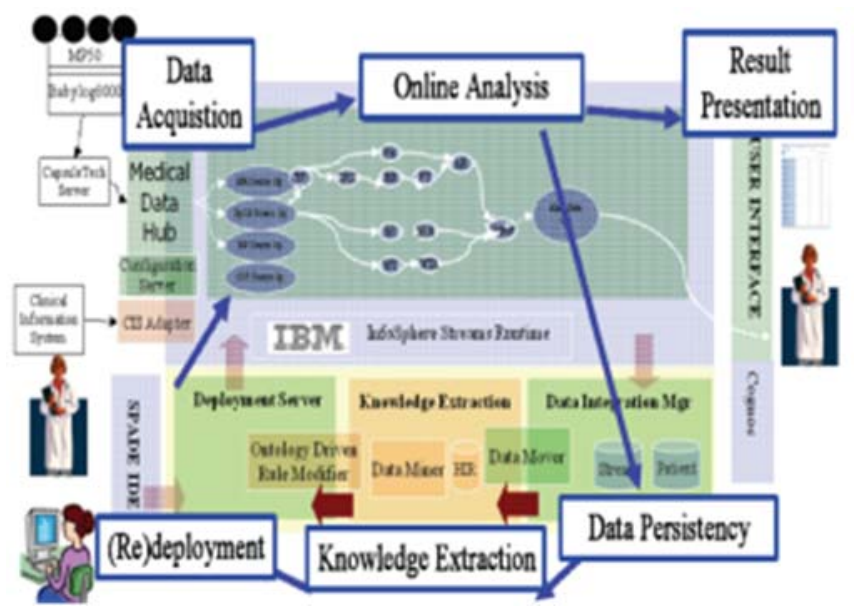

Figure 1. Artemis [4] Architecture

\subsection{Artemis Cloud}

Khazaei et al [8] has proposed a cloud-based Health Analytics as a Service (HAaaS) framework that enabled Artemis to be offered as an Artemis Cloud HAaaS. In that framework, the Data Acquisition component of Artemis can be located at a different location to the remaining Artemis components which are provisioned through cloud services. To facilitate this, a Data Transmission component was added to the framework. Artemis Cloud as a HAaaS was extended in [5] to employ an application program interface (API) within a Data Transformation component that processes data records within data streams when captured live from bedside monitors. In this approach, as shown in Figure 2, the API is a new component on the cloud computing architecture within Artemis Cloud which allows raw data streams to be processed as producers of medical data and then made available for other services as consumers of medical data. With this method, multiple processes can ingest the same data streamed out in parallel from multiple bedside monitors. Before providing the processed data for ingestion by other services, the API first takes multiple incoming data streams from a middleware that standardizes and structures the data. This approach allows smooth flow of data from incoming streams to analytical services. However, within that HAaaS approach the Data Acquisition and Data Transmission components are still tightly coupled within the overall HAaaS framework and instantiated Artemis platform. 


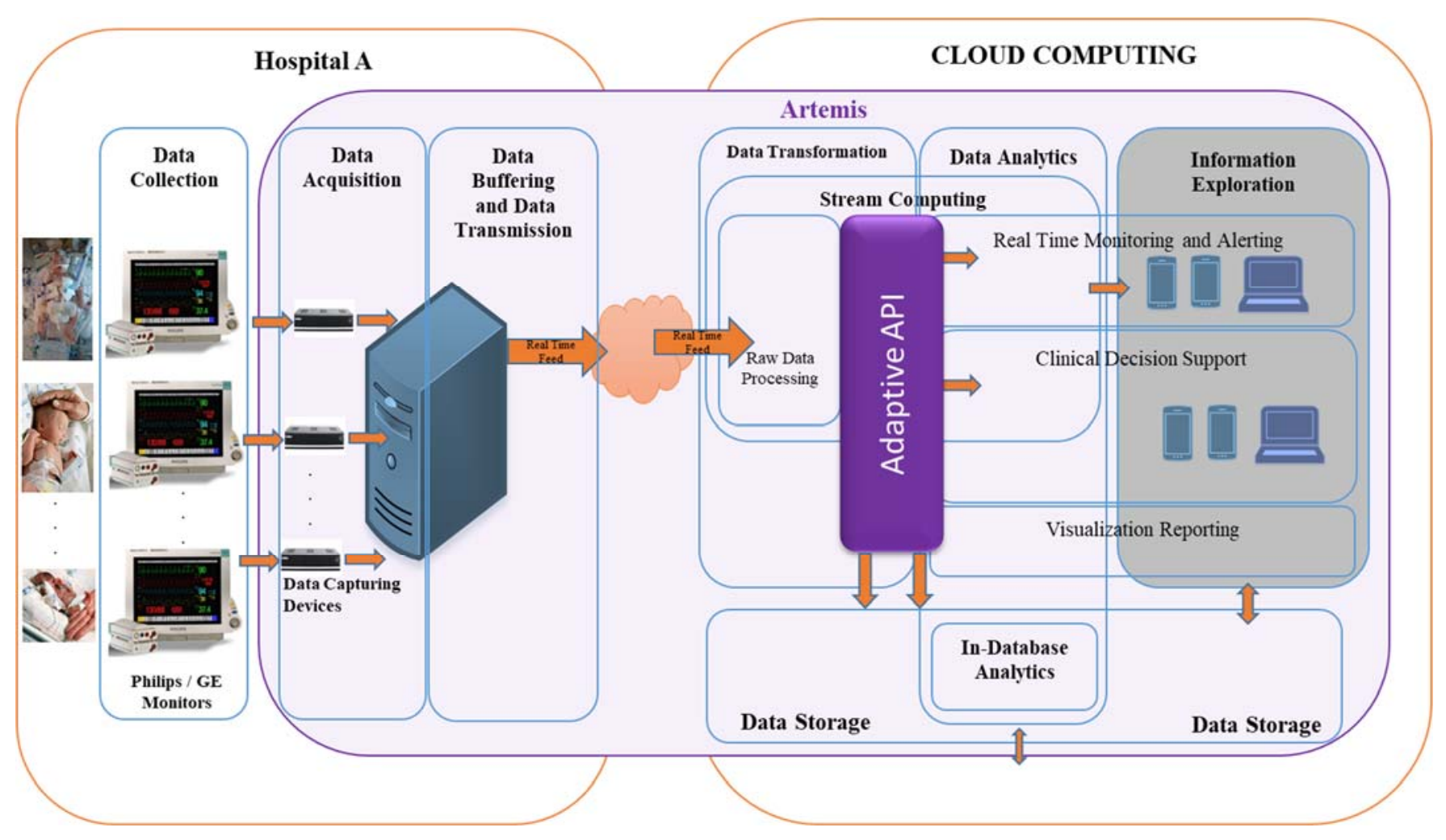

Figure 2. Artemis Cloud [3]

\section{DATA WAREHOUSING FRAMEWORK FOR LOW RESOURCE SETTING}

Yichuan Wang et al [9] in their study have proposed a generic best practice big data analytics architecture. That architecture is comprised of five major architectural layers: (1) data layer, (2) data transformation, (3) analytics, (4) information exploration and (5) data governance. However, that architecture does not support the analytics being provided as Health Analytics as a Service and decoupled from the other components.

This paper proposes an extended HAaaS framework that decouples the Data Collection and Data Acquisition components from the remaining components within a big data analytics data warehousing framework. Communication between the Data Acquisition and remaining components is enabled through Data Transmission and optional Data Buffering components. Figure 3 shows the proposed generic design that we propose for a decoupled HAaaS framework that we instantiated within Artemis cloud. As noted in the recent literature review [4], any such big data analytics framework would have all its components tightly integrated. There is a need to be able to decouple Data Collection, Data Acquisition and Data Transmission components from the remaining components that will be provided as a service. From a data warehouse/analytics/analytics as a service perspective, we need to decouple the Data Collection, Data Acquisition and Data Transmission components from the HAaaS that is being provisioned by Artemis Cloud.

The proposed big data analytics framework design is comprised of seven components: (1) data collection (2) data acquisition (3) data transmission, (4) data transformation; (5) data analytics; (6) information exploration/visualization and (7) data storage. In this framework, Data Collection, Data Acquisition and Data transmission have been decoupled from the remaining components. So, Data Collection, Data Acquisition and Data Transmission will take place within the Healthcare Organization and their design and installation can be managed independently of the remaining components. The collected data will be sent via the cloud to the Artemis Cloud deployment which will be ingested by the Adaptive API within the Data Transformation layer. The Data Analytics will be performed by the Artemis Cloud instance and the result will be sent back to the healthcare organization providing Health Analytics as a Service.

\subsection{Data Collection}

During data collection, a range of medical devices collect information from the patient via a range of sensors and transform that signal into high frequency data streams. These data streams are created at differing frequencies depending on the device. The high frequency data streams are displayed on the medical device as a univariant display. Some of these medical devices send these high frequency data streams to an output port that can be serial, Ethernet or USB. As the sensors continuously capture the baby's physiological features in the form of high frequency data streams at bedside and then display it on monitors, likewise is the flow of this data to Artemis Cloud instance. With Artemis cloud the architecture can capture and display on the monitors all the physiological features of the baby such as heart rate, respiratory rate, blood pressure, blood oxygen saturation and ECG waves [5]. The monitors are attached to a 'data integrator' or 'data socket' software that would capture the data in an electronic form and forward it to the local data warehouse located on a highperformance data server [10]. 


\subsection{Data Acquisition}

High frequency data streams that are produced via the monitoring devices and other equipment are collected through the data acquisition process. Any medical equipment has its own communication protocol so there is a need for software also known as a Data Socket or Data Integrator to extract information from the specific device. The data socket attaches a device ID which tells which device the data is being acquired from and a reference ID which tells which baby the data is referring to. The data Integrator software formats the data received into a form that can be transmitted as part of the high frequency data stream. The high frequency data stream supplied by each data socket may contain elements like Device ID, Indicator ID, Reference ID, Date/Time Stamp etc. Within this case study the baby's physiological data represents the high frequency data stream. The Device ID would identify the monitor or the device attached to the baby. Indicator ID would identify the reading types produced by the same device, for example, the bedside Pulse Oximeter monitor provides information about both baby's heart rate and oxygen saturation. The Reference ID would identify the baby which is attached to the device with a given Device ID. The Date/Time stamp would give information about when the reading occurred [10].

\subsection{Data Transmission (and optional Data Buffering)}

The data transmission component enables the transmission of the data as streams of data emanating from the medical devices in realtime. Both network outages along with maintenance outages at the cloud host site cause a less than $100 \%$ availability of the remaining Artemis Cloud components. Data loss can be eliminated through the use of an optional Data Buffering component that buffers the data when outages occur and sends the data at a high speed once the other components are accessible again, to facilitate a catch up to real-time processing once more. The physiological data received in the form of high frequency data streams is transferred to physiological base table within the Data Warehouse that would have a table structure that matches the format of the data received [10].

\subsection{Data Transformation}

Data transformation involves converting the transmitted data from the Healthcare Organization into a form that can be forwarded further for Data Analytics. This is enabled by the Adaptive API component of Artemis that process and transforms data from producers for use by consuming analytics and data storage processes within the Online Analytics component.

\subsection{Data Analytics}

Data analysis involves stream computing, which supports high performance stream data processing in near real-time or real-time. Real-time analysis allows tracking of data in motion, tracking of unexpected events as they happen and decision making.

\subsection{Information Exploration/Visualization}

Information exploration layer includes the visualization layer as shown in Figure 3. This layer is responsible for generating outputs such as various visualization reports or real-time information monitoring. Within the health care domain this layer provides time critical output, such as real-time monitoring of information for the generation of proactive real-time alerts, as well as, other visualizations to support clinical decision making for a range of neonatal conditions.

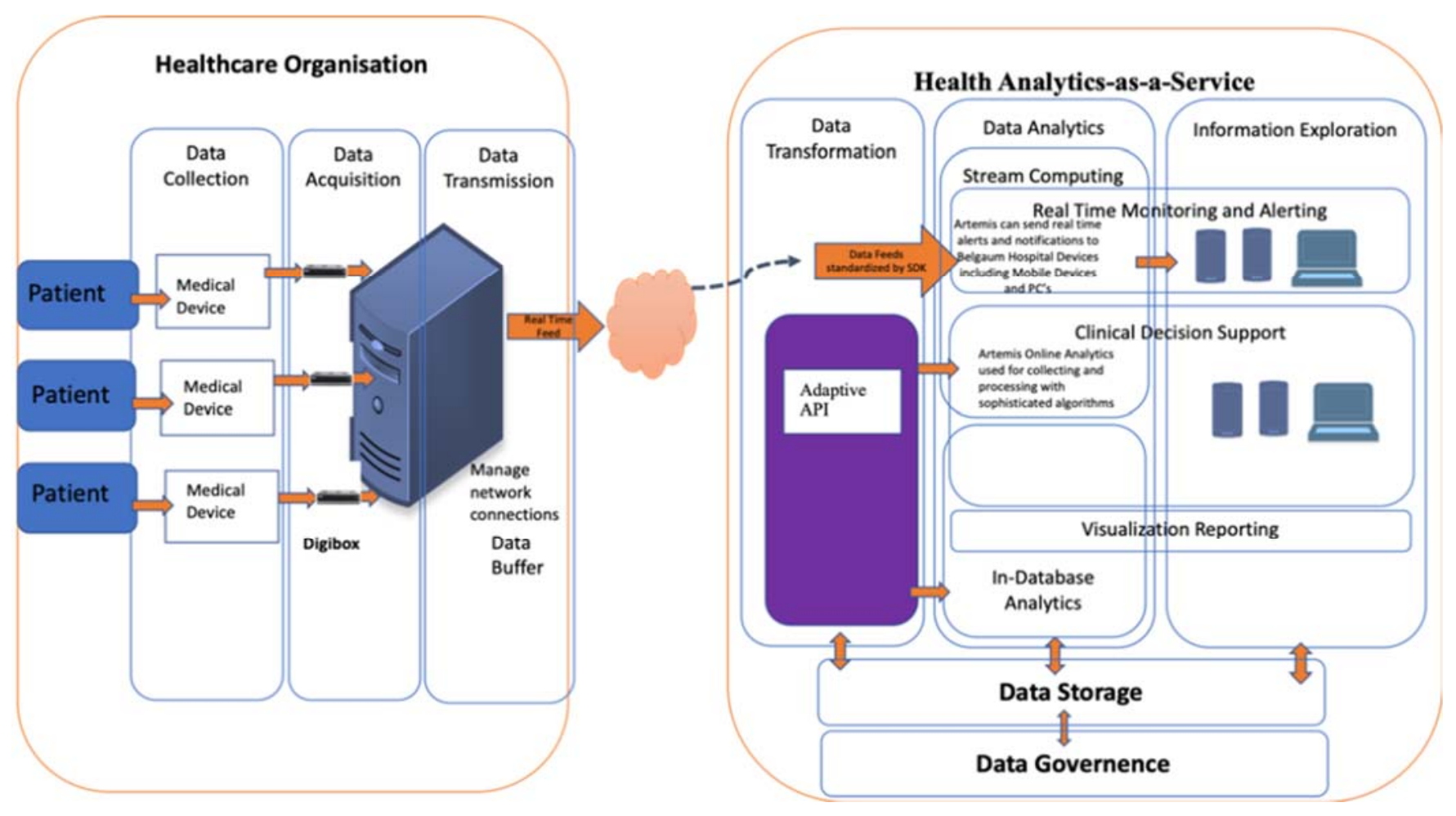

Figure 3. General Architecture of the Framework 


\section{CASE STUDY INSTANTIATION OF FRAMEWORK}

To demonstrate and test this approach we have setup the environment for our case study at Belgaum Children Hospital to enable Artemis Cloud to support critical care within their NICU. This is an appropriate case study for this research targeted for low resource setting environments, like $\mathrm{BCH}$.

$\mathrm{BCH}$ has an outborn NICU with 35 bed spaces. Roughly $40 \%$ of the babies are brought in with birth asphyxia and $50-60 \%$ are preterm. Premature babies within this NICU can be as early as 2830 weeks gestation. Another major reason for admission is jaundice. The average length of stay is 7-10 days and for babies with birth weight less than $1 \mathrm{~kg}$, it is more than 15 days. At $\mathrm{BCH}$, preterm infants receive ventilation $=$ assistance, as required, including the use of Variable Positive Airways Pressure (VPAP) and ventilators. There are no incubators. Instead, open care warmers are used.

We have proposed an initial deployment of a new decoupled HAaaS based Artemis Cloud for four bed spaces within the $\mathrm{BCH}$ NICU. A video walk through of the BCH NICU was prepared to capture the current layout and technical architecture within the NICU [11]. The platform is capable of gathering physiological data from bedside medical devices (pulse oximeter) at $\mathrm{BCH}$ and transferring it in a secure way to the Artemis cloud deployment at the Centre for Advanced Computing, Queen's University's Compute Ontario infrastructure via the cloud.

\subsection{Goals and Objectives}

This research proposes an initial end to end connection test from up to four bed spaces within the NICU, BCH.

\subsection{Data Collection}

Each neonatal patient at each NICU is connected to a Nellcor 595 medical device that is kept at that bed space location. Sensors are attached to the neonatal hand or foot to enable the Nellcor 595 to acquire the plethysmography waveform these devices generate from the sensor on the neonatal infant. The Nellcor device derives blood oxygen saturation levels and pulse rate from that waveform at a frequency of a reading every two seconds $(0.5 \mathrm{~Hz})$.

\subsection{Data Acquisition}

We will utilize the existing Nellcor 595 pulse oximeter monitors to enable us to collect pulse rate, and blood oxygen saturation at 0.5 $\mathrm{Hz}$ frequency. As such, instead of using the heart rate derived from the electrocardiogram signal within other Artemis deployments [5] we will use the pulse rate derived from the plethysmography wave, where each peak in the wave corresponds to one heartbeat. The derived blood oxygen saturation levels will also be used. A client server instance was created to facilitate the collection and transmission of the physiological data to the Artemis Cloud. The Nellcor device's serial port was connected to the serial port of a serial to Ethernet converter. This enables the converter to forward the Ethernet formatted signal to a 4-port switch. In addition to the physiological data collected from the Nellcor device, clinical data is manually collected from paper charts.

For this BCH case study, the Data Acquisition and Data Transmission integrator software was developed at Ontario Tech University, based on design specifications from the Indian based team members for use on a laptop. The devices were connected to the laptop for phase one testing and will be connected to a laptop located at $\mathrm{BCH}$ for the second phase of testing.

\subsection{Data Transformation}

The Nellcor 595 medical device data output via the serial port is configured by the device to be sent directly to a printer. The data format cannot be changed. A detail row for the printed report is generated every two seconds and where required for the paper report page header data packets are generated prior to the data packet to correspond with commencing of a new page. Therefore, data transformation is required to remove the header and footer data packets, so that only the data packets at the $0.5 \mathrm{~Hz}$ frequency are forwarded for data analytics.

\subsection{Data Transmission}

During the first phase of testing, data was transmitted from the Health Informatics Research laboratory at Ontario Tech University to the Artemis Cloud instance operating as a cloud Health Analytics as a service (HAaaS) located on a Compute Ontario cloud infrastructure, located at the Centre for Advanced Computing at Queen's University.

During the second phase of testing, data will be transferred from within the Belgaum Children's Hospital Neonatal Intensive Care Unit to the Artemis Cloud instances, operating as a cloud Health Analytics as a service (HAaaS), located on Compute Ontario cloud infrastructure located at the Centre for Advanced Computing, Queen's University. We will utilize a secure VPN tunnel for this transmission for security and privacy compliance. Device location will enable the data to be tagged with a device ID and this will be translated to a study identifier based on clinical information as to which neonatal infant is in that bed space at that time.

\subsection{Data Analytics}

We will perform data analytics on this data based on the principles of the analytics detailed in $[12,13]$ for the clinical conditions of LONS and RoP.

Research is expected to be completed in two phases to meet its objectives. The goal of phase one was to support initial end to end testing with the Nellcor monitor (bedside monitor at $\mathrm{BCH}$ ) not connected to any human. This testing confirmed the functionality of decoupled Artemis Cloud platform and demonstrated the viability of this decoupled framework. It enables this instance of Artemis Cloud to receive data from the test device. Performing the test in this way was advantageous as it did not require ethics approval or a clinical protocol for clinical data collection.

Phase two will be facilitated through a clinical research study, where up to four Nellcor devices attached to the newborn infants in the NICU BCH will be connected to this new Artemis Cloud decoupled infrastructure, to support a clinical research study that will operate as a parallel test to compare what alerts were generated by Artemis Cloud with clinical practice suspicion and detection of these conditions. As a result, the Information Exploration component will not be deployed in these first two phases. In future work, we will assess the functionality and usability of the information exploration component at the bedside.

\section{RESULTS}

This section presents the results of the creation of an instance of the new decoupled framework proposed in this paper that extends the Artemis Cloud platform to enable the decoupling. The case study for the testing of this alternate architecture is the NICU Belgaum Children's Hospital.

The goal of this testing was to demonstrate the viability of this decoupled approach for HAaaS. In order to test this decoupled 
approach for HAaaS, we established a testing architecture as shown in Figure 4.

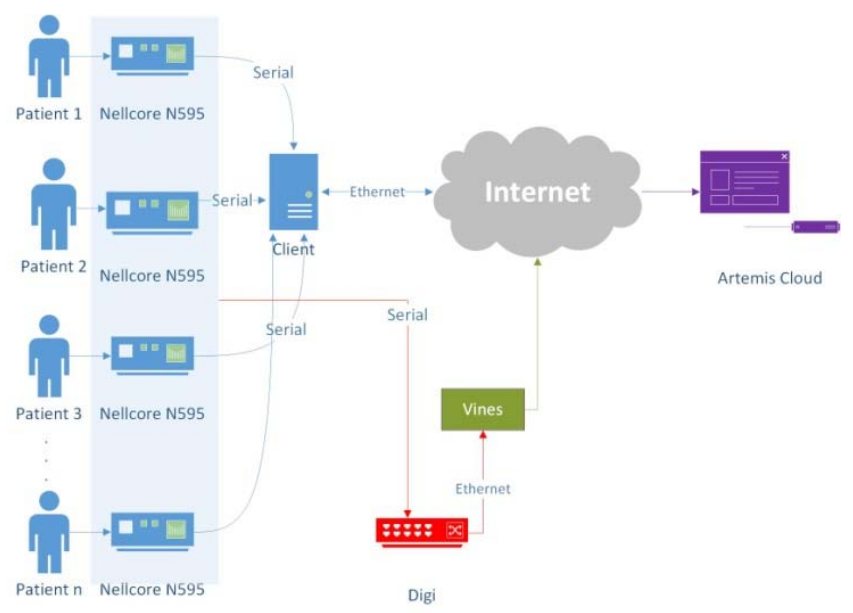

Figure 4. Testing Architecture to Test Decoupled Approach for HAaaS

The Nellcor 595 devices used in the NICU, Belgaum Children's Hospital are shown in blue in Figure 4 along with the newborn infants in the NICU that they are connected to. The previously architected method for Data Acquisition and Data Transmission that are tightly coupled within the overall Artemis architecture are the Digi serial to Ethernet convertor in red and the Vines software in Green in Figure 4. Digi is a device that contains multiple serial ports and it allows retrieval of data from multiple medical devices at the same time. It converts serial data to TCP and enables the transferring of that data via Internet to Artemis Cloud. The Vines server is used for buffering and queue management. This enables data to be buffered and then sent at a faster than regular rate in the event of a network or other component outages. To provide this solution for Data Acquisition and Data Transmission requires an approximate \$US1500 investment per bed space. A decoupled approach for Data Acquisition and Data Transmission as proposed in this research is shown as the client in blue. Within this testing architecture, an instance of Artemis Cloud was created in the testing environment of Artemis Cloud physically located in the Centre for Advanced Computing, Queen's University. This instance of Artemis Cloud is denoted in the purple within Figure 4.

This paper reports on the first stage testing. The second stage testing will be reported as part of the results of the clinical study in a future publication. To perform this test, the Nellcor 595 device was utilized that was located within the Health Informatics Research Laboratory at Ontario Tech University. The device was in operation but not connected to any humans. This allowed the device to transmit data packets at its usual rate of $0.5 \mathrm{~Hz}$. The data packets were the appropriate structure but had invalid data in the measurement fields. This invalid data did not impact the function of the testing. To perform all the functionality required within the new decoupled Data Acquisition component, a Python script was developed for the client which is responsible for retrieving data from the medical device via the serial port, encapsulating that data into the TCP packets and transferring the data to the remote server by establishing the TCP connection. The Artemis Adaptive API is utilized to receive the data from the decoupled Data Acquisition component. As a result, this data becomes a new producer for the Artemis Adaptive API. Data Analytics consumers for LONS and
RoP that utilize blood oxygen saturation and or pulse rate at a frequency of $0.5 \mathrm{~Hz}$ or slower can subscribe to and consume this data as output by the Adaptive API. The architecture as described above and shown in Figure 4 was enacted for testing purposes. Discontinuous testing sequences were performed over 3 weeks. The Python script successfully acquired and transmitted all packets of data received from the Nellcor device during the testing. Receipt of the data by Artemis Cloud was impacted by network reliability as the client developed for this test did not contain components for buffering.

The raw signal from the Nellcor device generates up to 128 bytes of data every two seconds for the data packets and 3 sets of 128 bytes for 3 lines of header rows that are generated for each new page in preparation for paper printing. Each of the four connected Nellcor devices will generate this volume of data when connected to a neonatal infant. This data must then be wrapped in additional messaging for transmission over Ethernet.

\section{DISCUSSION}

Wang et al [9] in their study define big data analytics capability within healthcare domain as the ability to acquire, store, process and analyze large amount of health data which could be in structured or unstructured or semi structured form, and deliver meaningful information to users which in turn would allow them to discover business values and insights at right time. The authors have proposed a big data analytics architecture which is based on the concept of data life cycle framework that would start with data capture, proceed with data transformation and end with data consumption. The $\mathrm{BCH}$ case study discussed in this paper also incorporates the same idea of big data analytics architecture following the concept of data life cycle framework comprising of five major architectural layers, which are data (data collection, data acquisition), data aggregation (data transmission, data transformation), data analytics, information exploration and data governance.

Artemis cloud is a powerful analytical center for health data and serves as a tightly integrated real-time platform to deliver secure and timely critical care. Physiological data of patients that are connected to the web-enabled open network can be transmitted in the form of synchronous streams and can be consumed by the event stream engine of Artemis cloud providing an insight to patient's dynamic health status for specialists located in care centers. Medical devices within the BCH NICU can be connected using a thin client model as proposed in this paper enabling secure transmission of physiological data streams through the physiological web service component of the Artemis cloud. Kamaleswaran R et al [14] have discussed some stable connection issues using Artemis cloud instance to detect events in real-time that need to be addressed.

Throughput would be variable depending on the load of the network infrastructure locally as well as remote. Variable throughput will result in timeout to the connection, requiring the Artemis client system at Ontario Tech's deployment at the Centre for Advanced Computing at Queen's University to reinitiate the session. As the client cannot be configured automatically, when the sessions terminated it would require physical intervention. To address these limitations with Artemis cloud use of HAaaS paradigm and IoT would be a better solution [14]. Inibhunu et al proposed an extended version of Artemis Cloud that provides the ability to connect via an API but to date they have demonstrated it within a tightly coupled context at McMaster Children's Hospital, Ontario Canada and Southlake Regional Health, Ontario, Canada. 
Those deployments use Vines for connection to the medical devices at the bedside for Data Acquisition. Vines software is tightly coupled within Artemis Cloud. Opportunities exist to utilize other forms of Data Acquisition and forward the data to the Artemis API in a loosely coupled framework [3]. This paper presents an alternate approach to Data Acquisition that is not tightly coupled with Artemis Cloud.

\section{CONCLUSION}

This paper has presented an extended Data Warehouse framework that allows decoupling of data collection and acquisition components from the other components of the data warehouse framework within a Big Data Analytics Data Warehousing framework. This has been instantiated within an extended Artemis Cloud platform.

Creating a Data Acquisition component requires an understanding of the output protocol from the medical device. Knowledge of these protocols is difficult to obtain for devices such as the Philips Intellivue series monitors and the GE Dash Monitors. Hence, the prior versions of the Big Data analytics data warehouse framework and Artemis Cloud instantiation used software such as Vines for device connectivity within. Newer standards such as the IEEE 11073 Medical Device standard promote a landscape of open and standardized data structures for data exchange that will further support the decoupled approach proposed in this article.

The Data Acquisition component proposed within this approach did not support buffering and queuing management of data. As such, network outages and maintenance windows on virtual machines where the Artemis Cloud platform resides directly impact whether data will be made available to Artemis for analysis. This will be addressed in future research.

The extended Artemis Cloud platform has been demonstrated through a simulated test between Ontario Tech University and the Centre for Advanced Computing, Queen's University as part of Compute Ontario infrastructure. The proposed framework allows decoupling of components within the framework using HAaaS paradigm; this approach is not seen within any other existing frameworks reviewed in the literature. The case study presented in this paper and associated prototype provides a mechanism to validate the application of this architecture to Neonatal Intensive Care Units.

\section{ACKNOWLEDGMENTS}

We would like to thank Dr Arun Desai and Dr Anand Karkal at the Belgaum Children's Hospital for their extensive help and support with this research. This research is supported by McGregor's Canada Research Chairs Program grant (No 950-225945), McGregor's Research Excellence Chair grant, the Canadian Foundation for Innovation (No 203427) and the Centre for Advanced Computing, Queen's University as part of Compute Ontario.

\section{REFERENCES}

[1] McGregor C, James A, Eklund M, Sow D, Ebling M, Blount M, (MEDINFO 2013), "Real-time Multidimensional
Temporal Analysis of Complex High Volume Physiological Data Streams in the Neonatal Intensive Care Unit"

[2] Balaji, S., Patil, M., McGregor, C., 2017 “A Cloud based Big Data Based Online Health Analytics for Rural NICUs and PICUs in India: Opportunities and Challenges", 30th International Symposium on Computer-based Medical Systems (CBMS 2017), pp 385-390

[3] McGregor C. A cloud Computing Framework for Real-time Rural and Remote Service of Critical Care.

[4] Bastwadkar, McGregor, Balaji "Trends and Opportunities in Health Analytics as a Service and Implications for Use in Low Resource Settings: A Literature Review", Technical Report, Jain University, 2019

[5] Inibhunu, C., Jalali, R., Doyle, I, Gates, A., Madill, J., McGregor, C., 2019, "Adaptive API for Real-Time Streaming Analytics as a Service", $41^{\text {st }}$ IEEE Engineering in Medicine and Biology conference, Berlin, Germany, pp34727

[6] McGregor C 2013. Big Data in Neonatal Intensive Care, IEEE Computer, 46(6): 54-9

[7] McGregor C 2013. Big Data in Neonatal Intensive Care, IEEE Computer, 46(6): 54-9

[8] Khazaei, H., McGregor, C., Eklund, J.M., El-Khatib, K., 2015, "Real-time and Retrospective Health Analytics as a Service”, JMIR Medical Informatics, 3(4), e36

[9] Wang, Y., Kung, L., \& Byrd, T. A. (2018). Big data analytics: Understanding its capabilities and potential benefits for healthcare organizations. Technological Forecasting and Social Change, 126, 3-13.

[10] McGregor C 2002, Bryan G, Curry J,Tracy M. The e-Bay Data Warehouse: A Case Study. Proceedings of the 35th Hawaii Internaional Conference on System Science - 2002.

[11] https://youtu.be/KJiAoMfK-co. 11-Jan-2016 at 5:36AM.

[12] McGregor, C., Catley, C., James, (2012), "Variability Analysis with Analytics Applied to Physiological Data Streams from the Neonatal Intensive Care Unit", 25th IEEE International Symposium on Computer-Based Medical Systems (CBMS 2012), Rome, Italy, CDROM 5 pages

[13] Fernando, K.E.S., C. McGregor, C., James, A.G., 2016, "Correlation of Retinopathy of Prematurity and Blood Oxygen Saturation in Neonates using Temporal Data Mining: A Pilot Study", 38th Annual International Conference of the IEEE Engineering in Medicine and Biology Society, Orlando, USA

[14] Kamaleswaran, R., Thommandram, A., Zhou, Q., Eklund, M., Cao, Y., Wang, W. P., \& McGregor, C. (2013, June). Cloud framework for real-time synchronous physiological streams to support rural and remote Critical Care. In Proceedings of the 26th IEEE International Symposium on Computer-Based Medical Systems (pp. 473-476). IEEE. 


\section{Columns on Last Page Should Be Made As Close As Possible to Equal Length}

\section{Authors' background}

\begin{tabular}{|l|l|l|l|}
\hline Your Name & Title* & Research Field & Personal website \\
\hline $\begin{array}{l}\text { Meghana } \\
\text { Bastwadka } \\
\text { r }\end{array}$ & $\begin{array}{l}\text { Research } \\
\text { Scholar }\end{array}$ & $\begin{array}{l}\text { Computer } \\
\text { Science }\end{array}$ & \\
\hline $\begin{array}{l}\text { Carolyn } \\
\text { McGregor }\end{array}$ & $\begin{array}{l}\text { Full } \\
\text { Professo } \\
\text { r }\end{array}$ & $\begin{array}{l}\text { Computer } \\
\text { Science, } \\
\text { Health } \\
\text { Informatics }\end{array}$ & $\begin{array}{l}\text { https://businessandit.ontariotechu.ca/people/faculty/manageme } \\
\text { bappsc-smieee.php }\end{array}$ \\
& $\begin{array}{l}\text { Full } \\
\text { Professo }\end{array}$ & $\begin{array}{l}\text { Computer } \\
\text { Science } \\
\text { and } \\
\text { Engineerin } \\
\text { g Balaji }\end{array}$ & $\begin{array}{l}\text { https:/navigator.innovation.ca/en/facility/ontario-tech- } \\
\text { university/joint-research-centre-ai-health-and-wellness }\end{array}$ \\
\hline
\end{tabular}

*This form helps us to understand your paper better, the form itself will not be published.

${ }^{*}$ Title can be chosen from: master student, Phd candidate, assistant professor, lecture, senior lecture, associate professor, full professor 